\title{
PRÉSENCE DE LA CREVETTE : ATYAEPHYRA DESMARESTI MULLET AUX ENVIRONS DU PARACLET (SOMME)
}

\author{
par A. WURTZ
}

La petite Crevette d'eau douce : Atyaëphyra desmaresti Millet ne peut plus être considérée comme une rareté, car on en connaît déjà un certain nombre de stations en France. Dans un article paru dans ce bulletin en 1943, M. ANDRÉ (1) signalait un certain nombre de stations dans l'Ouest (Anjou, Bretagne) où elle est parfois assez fréquente, dans l'Est (canal de la Marne au Rhin, à Nancy, en Alsace) dans la Marne près de Paris (Alfort, Créteil) et dans le canal de Saint-Maurice. Elle est assez abondante en Belgique où elle paraît vivre exclusivement dans les canaux et les rivières canalisées.

Il me paraît néanmoins intéressant de signaler la présence de ce joli Crustacé aux environs de la Station d'Hydrobiologie du Paraclet, non pas pour ajouter une station de plus à celles déjà connues - ce qui en soi ne présente pas un intérèt particulier - mais pour préciser quelques points de' son écologie.

Les deux endroits où Atyaëphyra a été trouvée sont en effet nettement différents. En Mai 1949, j'en ai trouvé quelques individus dans les étangs du Marais de Pavery à 1 kilomètre environ à l'Est du lieudit a Pont de Fouencamps ". En Mars 1950, un coup de filet-troubleau effectué par le garde-pêche Peltier dans l'Avre, rivière à courant assez rapide, a ramené un autre individu de très belle taille (longueur depuis le rostre jusqu'au telson : $36 \mathrm{~mm}$.)

Ajoutons simplement quelques précisions sur ces deux stations opposées.

Les étangs du Marais de Pavery sont d'anciens étangs d'exploitation de tourbe (tourbières à Hypnacées), à $\mathrm{pH}$ alcalin, assez riches en chaux; les poissons dominants sont des Brêmes, Brochets, Gardons, Rotengles, Carpes. L'eau de ces étangs peut se réchauffer assez fortement en été et sa température monter jusqu'à $25^{\circ}$. $A$. desmaresti se tient normalement dans les herbiers de Potamogeton natans, Ranunculus aquatilis, Cerato-

(1) ANdre (M.). - Sur une Crevette habitant nos eaux douces : Atyaëphyra desmaresti Millet, Bull. Franç. de Pisciculture, no 129, 1943, pp. 132-136. 
phyllum et Myriophyllum, sur le bord des étangs. Le fond est généralement assez fortement envasé et recouvert de feuilles mortes de peupliers ou de saules en décomposition. Les animaux accompagnant notre Crevette, récoltés au filet-troubleau (larves d'Insectes : Sialis, Agrion, etc. ; Insectes : Nepa cinerea; Crustacés : Aselles; Mollusques : Limnées, Sphaerium corneum, Planorbis carinatus) indiquent d'ailleurs bien une eau stagnante, assez riche en matières organiques en décomposition.

Dans l'Avre au contraire $A$. desmaresti vit nettement en eau courante. Au niveau du "Pont de Fouencamps ", le courant est même assez fort. Mais il faut reconnaître que la Crevette se tient dans les herbiers de Ranunculus fluitans, Helosciadium nodiflorum, dans des endroits où le courant est brisé. La température de l'eau, qui était de $7^{\circ}$ en Mars, peut descendre jusqu'à $3^{\circ}$ ou $4^{\circ}$ en hiver. Les animaux récoltés au mème endroit (très nombreuses larves de Simulies sur les touffes de Renoncules, larves d'Éphémères non déterminées, très nombreux Mollusques : Neritina fuviatilis sur les cailloux immergés, ainsi que des Éponges : Spongilles) et les Algues rhéophiles : Vaucheria, Chaetophora, Ulothrix, qui couvrent abondamment les pierres, montrent qu'il s'agit d'une station d'eau courante froide nettement différente de la précédente.

Nous savions déjà que ce petit Crustacé, bien que d'origine méridionale, était très eurytherme et supportait des variations assez fortes de température; nous savions aussi par ailleurs que, bien qu'acclimaté depuis longtemps en eau douce, il pouvait supporter des variations de salinité.

La trouvaille d'Atyaëphyra desmaresti aux environs du Paraclet, dans des stations géographiquement très voisines, mais aussi différentes que l'eau stagnante d'étang et l'eau courante de rivière froide, apporte donc une preuve de plus que cette espèce est eurytherme et capable de vivre dans des milieux écologiques assez différents. 